\title{
Data-driven Modeling and Real-time Distributed Control for Energy Efficient Manufacturing Systems
}

\author{
Jing Zou ${ }^{\mathrm{a}}$, Qing Chang ${ }^{\mathrm{a}, *}$, Jorge Arinez ${ }^{\mathrm{b}}$, Guoxian Xiao ${ }^{\mathrm{b}}$ \\ ${ }^{a}$ Dept of Mechanical Engineering, Stony Brook University, Stony Brook, New York 11794 \\ ${ }^{b}$ General Motors R\&D, General Motors Corporation, Warren, MI 48090
}

\begin{abstract}
As manufacturers face the challenges of increasing global competition and energy saving requirements, it is imperative to seek out opportunities to reduce energy waste and overall cost. In this paper, a novel data-driven stochastic manufacturing system modeling method is proposed to identify and predict energy saving opportunities and their impact on production. A real-time distributed feedback production control policy, which integrates the current and predicted system performance, is established to improve the overall profit and energy efficiency. A case study is presented to demonstrate the effectiveness of the proposed control policy.
\end{abstract}

Keywords: Energy control methodology, data-driven modeling, system diagnosis and prognosis, feedback control, distributed control, Energy profit optimization

\section{Introduction}

Conventionally, improving production efficiency, flexibility and responsiveness has been the primary research focus of production management, while energy consumption has received little attention. As concerns mount about long-term energy availability and climate change, energy consumption and energy efficiency improvement are becoming companies' top issues.

To achieve the goal of reducing energy use from manufacturing plants by twenty percent to fifty percent for the next three to ten years [1], critical technical challenges must be solved. First, there is a lack of rigorous modeling method for real-time system level monitoring, identification and prediction of energy waste and energy saving opportunities. While information about production systems has becoming increasingly transparent, detailed, and real-time, the utilization of this information for energy efficiency improvement and cost reduction has been lagging behind. The traditional methodologies for production system analysis and control are mostly based on steady state analysis and long-term performance measurements. Hence, the advantage of detailed sensor data, which is critical to improve responsiveness of the real-time system control, is mostly ignored. The vast amounts of time series data generated by distributed sensors have to be analyzed in real-time and converted to actionable knowledge that can be fed to process monitoring, control, and optimization algorithms [1]. The challenge now is to disseminate and interpret the information collaboratively through data-driven modeling for intelligent control such that the entire plant floor operations and energy consumption are well coordinated, connected, and timely reconfigured with the goal of achieving higher energy efficiency and sustainability for the entire system.

Second, there is a lack of real-time intelligent control method for manufacturing energy optimization. There is a long history of research in control theory but the manufacturing domain has not in general been a

\footnotetext{
* Corresponding author. Tel.: +1 631-632-8329; fax: +1 631-632-8544.

E-mail address: qing.chang@stonybrook.edu.
} 
target for this research [1]. Consequently, most of the current energy optimization and control methods focus on heuristic rules or require computationally expensive time horizon algorithms, such as dynamic programming algorithm, where an optimal solution is obtained through an exhaustive numerical search. This will seriously hinder the effectiveness of the control in real-time advanced manufacturing environment. Due to the stochastic and non-linear properties of production systems, it is a big challenge to establish a control policy to enable a fast response to random disturbances and unexpected changes with the goal of improving overall economic benefit and energy efficiency.

This paper is devoted to address the above challenges. The main contributions of this paper are in: 1) establishing a data-driven mathematical model through available sensor data; 2) developing a system diagnosis and prognosis methodology based on the data-driven model to identify and predict the system performance and energy saving opportunities; 3) establishing a novel automated distributed control scheme which integrates real-time feedback control and collaborated control to improve overall system profit and energy efficiency.

The rest of the paper is organized as follows: literature review is provided in Section 2. Section 3 introduces the assumptions and notations applied in the paper. In Section 4, a mathematic model for a continuous flow stochastic production system is developed. Energy saving opportunity and system performance identification and prediction are discussed in Section 5. Section 6 describes the automated distributed control scheme based on the stochastic model of the production system. Section 7 studies the energy and production economics of the manufacturing system. A case study is presented in Section 8. Finally, conclusions and future research are given in Section 9.

\section{Literature Review}

The existing production management studies are mostly focused on improving production efficiency, flexibility, and responsiveness [2]-[9]. There have been substantial research efforts in the analysis of the production system dynamics and performance evaluation [10]-[16]. Both simulation and analytical methods are utilized to study the properties of production system. Computer simulation is known to be a useful tool in analyzing the dynamics of production system. However, it may suffer from long model development time and intense computational effort to obtain useful conclusions. For analytical methods, exact analytical results only exist for the two-machine-one-buffer system and systems with infinite buffer capacity or without buffers [10], [11]. Decomposition and aggregation methods are utilized with Markov chain models to estimate the performance of longer lines and assembly systems [10], [12]. However, most of the existing analytical studies evaluate the system steady-state performance and make optimizations on long-term production system schedule plans [10], [12], [19]. Chen et al. derive the mathematical model and closed-form expressions for transient performance evaluation of synchronized serial production lines with geometric machines [20]. However, the method only provides some basic properties such as settling time monotonicity, while the transient throughput and work-in-process are actually derived based on approximation.

Recently, with the wide adoption of distributed sensors in production systems, more real-time detailed sensing information is available that is potentially very useful in identifying system real-time performance [13], [15]-[18], [21], [22]. Analysis of downtime impacts and the cost of disruption incidents for multi-stage manufacturing systems are studied in [13] and [16]. Reference [21] develops a real-time disruption recovery model for two-stage production-inventory systems to obtain the optimal recovery plan based on the real-time influence of the system disruptions. However, these studies mainly focus on real-time system diagnosis without further prognosis for potential system performance in future time. A system identification method is desired to provide quick and accurate diagnosis and prognosis for system performance.

There is an increasing interest in the research of improving energy efficiency in manufacturing plant floor operations [23]-[30]. Previous efforts in this area mainly focus on isolated or mutually independent machines or processes [23]-[26]. Schudeleit et al. compare the general energy efficiency test methods (i.e., reference 
part method, reference process method, specific energy consumption method, and component Benchmark method) for testing machine tools' energy efficiency using a multiple criteria decision-making technique and find the most promising candidate for ISO14955 series [25]. However, the following property is shared in modern manufacturing systems: the system exhibits highly complex dynamics since the operation status of each machine is determined not only by itself, but also by other machines and buffers. Therefore, it is indispensable to consider the interactions within production systems for effective energy management.

Energy optimization and control methods have also been developed and applied to improve energy efficiency at the system level [27]-[31]. Deif et al. focus on a system model for the new green manufacturing paradigm, which captures various planning activities to migrate from a less green into a greener and more eco-efficient manufacturing [27]. Reference [31] proposes a system approach for time-of-use based electricity demand response for sustainable manufacturing systems under the production target constraint. It is noted that these efforts are more focused on heuristic rules or expert knowledge due to the lack of mathematical theory and computational implementations that are sufficiently flexible and robust to deal with the complex nature of production systems.

Conventionally, researchers try to treat the production system as one process or system to be controlled. Due to the difficulty of a single central controller to deal with production system complexity, one widely used solution has been to distribute decisional capabilities to decisional entities, leading to non-centralized control system [32]. The control architecture is semi-heterarchical or fully heterarchical, and the control decision has been distributed to local entities. Chung et al. develop a modified genetic algorithm approach to deal with the distributed scheduling models with maintenance consideration, aiming to minimize the makespan of the jobs [33]. Reference [34] proposes a domain-based factory loading allocation problem for the conceptualization of a multi-site manufacturing supply chain, and a decision propagation structure incorporating with a connectionist approach is developed based on the concept of constraint heuristic search to facilitate the exploration of solution spaces. A systematic control scheme based on mathematical model is much desired.

\section{System description}

Continuous flow models are used in this paper because the production dynamics can be conveniently described by integral or differential equations [10], [16]. Continuous flow models assume the quantity of jobs in the buffer varies continuously from zero to its capacity. Consider a serial production line consisting of $M$ machines (represented by the rectangles) and $M-1$ buffers (represented by the circles) as shown in Fig. 1 . The following notations are used in this paper:

\section{Insert Fig. 1 about here}

- $\quad S_{i}$ denotes the $i^{\text {th }}$ machine, where $1 \leq i \leq M$;

- $\quad B_{i}$ denotes the $i^{t h}$ buffer, where $2 \leq i \leq M$;

- $\quad s_{i}(t)$ denotes the actual processing speed of machine $S_{i}$ at time $t$;

- $b_{i}(t)$ denotes the buffer level of buffer $B_{i}$ at time $t$;

- $K_{i}$ denotes the power rating of machine $S_{i}$;

- $\quad c_{p}$ denotes the profit per part produced;

- $c_{e}$ denotes the electricity rate;

- $c_{i}$ denotes the inventory cost per part per day;

- $\vec{e}_{i}=\left(j, t_{i}, d_{i}\right), i=1,2, \ldots n, j=1,2, \ldots, M$, represents a disruption event that machine $S_{j}$ is down at time $t_{i}$ for $d_{i}$ time period;

- $\quad \boldsymbol{E}$ denotes a sequence of disruption events, i.e., $\boldsymbol{E}=\left[\vec{e}_{1}, \ldots, \vec{e}_{n}\right]$; 
- $\quad M T B F_{i}$ and $M T T R_{i}$ denote the Mean-Time-Between-Failure and the Mean-Time-To-Repair of machine $S_{i}$ respectively. $\lambda_{i}=1 / M T B F_{i}$ and $\mu_{i}=1 / M T T R_{i}$;

- $P L_{\vec{e}_{i}}$ denotes permanent production losses attributed to disruption event $\vec{e}_{i}$;

- $P L_{\vec{e}_{i}}[a, b]$ denotes permanent production losses attributed to disruption event $\vec{e}_{i}$ during a time period $[a, b]$;

- $\quad P L_{i}$ denotes permanent production losses attributed to machine $S_{i}, 1 \leq i \leq M$;

- $P L_{i}[a, b]$ denotes permanent production losses attributed to machine $S_{i}, 1 \leq i \leq M$, during a time period $[a, b]$;

- $\quad P L$ denotes permanent production losses of the whole line;

- $\quad P L[a, b]$ denotes permanent production losses of the whole line during a time period $[a, b]$.

We make the following assumptions:

1) Each machine has a rated speed (or flow rate) $1 / T_{i}, i=1, \ldots, M$, where $T_{i}$ is the base cycle time of machine $S_{i}$

2) A machine will run at its rated speed if it is operational and is neither starved nor blocked;

3) $S_{M_{k}^{*}}$ denotes the slowest machines, i.e. $M_{k}^{*}=\arg \max _{i=1, \ldots, M}\left(T_{i}\right), 1 \leq k \leq M$;

4) $S_{M^{*}}$ denotes the slowest machine that is closest to the end-of-line machine $S_{M}$, since there might be one or multiple slowest machines in a system as described in 3). It is also denoted as the last slowest machine;

5) Each buffer $B_{2}, B_{3}, \ldots B_{M}$ has a finite capacity. With abuse of notation, $B_{2}, B_{3}, \ldots B_{M}$ are also used to denote the maximum capacity of the buffer;

6) A machine is starved if it is operational and its upstream buffer is empty and its upstream machine is down;

7) A machine is blocked if it is operational and its downstream buffer is full and its downstream machine is down;

8) The first machine $S_{1}$ is never starved and the last machine $S_{M}$ is never blocked;

9) Each machine $S_{j}$ will run at its power rating $K_{j}$ when up and will consume no power when turned off;

10) The machine warm up time is not considered for the ease of math expression;

11) The expenses to produce the part in terms of material and labor are already considered in the profit per part $\left(c_{p}\right)$ calculation.

\section{Data-driven mathematical model}

A manufacturing system is a stochastic dynamic system. The dynamic behavior of a stochastic system is usually modeled by state space equation of the form [35]

$$
\dot{\boldsymbol{X}}(t)=\boldsymbol{F}(\boldsymbol{X}(t), \boldsymbol{U}(t), \boldsymbol{W}(t))
$$

where $\boldsymbol{X}(t) \in R^{n}$ is the states, $\boldsymbol{U}(t) \in R^{m}$ is the known inputs or control, and $\boldsymbol{W}(t)$ is the unknown disturbance or noise at time $t$. Usually there is also an output $\boldsymbol{Y}(t) \in R^{l}$ modeled by the measurement equation

$$
\boldsymbol{Y}(t)=\boldsymbol{H}(\boldsymbol{X}(t), \boldsymbol{V}(t))
$$

where $\boldsymbol{Y}(t)$ is the observation or output and $\boldsymbol{V}(t)$ is the unknown measurement errors or noise.

According to [35], we have the following definition.

Definition 1: A stochastic system is given by specifying 
a) The state equation $\boldsymbol{F}(*)$ and the observation equation $\boldsymbol{H}(*)$, and

b) The joint probability distribution of the random variables in $\boldsymbol{W}(t)$ and $\boldsymbol{V}(t)$.

The measurement error or noise is not considered in this paper. Eq. (2) can be rewritten as

$$
\boldsymbol{Y}(t)=\boldsymbol{H}(\boldsymbol{X}(t))
$$

The manufacturing system equipped with sensor data is then described by Eqs. (1) and (3). The physical meanings of $\boldsymbol{X}(t), \boldsymbol{U}(t), \boldsymbol{W}(t)$, and $\boldsymbol{Y}(t)$ are:

$\boldsymbol{X}(t)=\left[X_{1}(t), X_{2}(t), \ldots, X_{M}(t)\right]^{\prime}$, where $X_{i}(t)$ is the production count of machine $S_{i}$ at time $t ;$

$\boldsymbol{F}(*)=\left[f_{1}(*), f_{2}(*), \ldots, f_{M}(*)\right]^{\prime}$, where $f_{i}(*)$ represents the dynamic function of machine $S_{i}$;

$\boldsymbol{U}(t)=\left[u_{1}(t), u_{2}(t), \ldots, u_{M}(t)\right]^{\prime}$, where $u_{i}(t)$ is the input or control at machine $S_{i}$ at time $t$. In this paper, a control of switching on or off a machine is adopted, which can be alternatively seen as speeding up and slowing down the machine. $u_{i}(t)$ is denoted by the binary variable as

$$
u_{i}(t)= \begin{cases}0, & \text { turn off machine } S_{i} \text { at time } t \\ 1, & \text { turn on machine } S_{i} \text { at time } t\end{cases}
$$

$\boldsymbol{W}(t)=\left[W_{1}(t), W_{2}(t), \ldots, W_{M}(t)\right]^{\prime}$, where $W_{i}(t)$ is the unknown random variable describing whether machine $S_{i}$ suffers from a disruption event at time $t . W_{i}(t)$ is directly related to $\boldsymbol{E}$, which can be obtained from sensor data. If $\exists \vec{e}_{k} \in \boldsymbol{E}$, s. $t . \vec{e}_{k}=\left(i, t_{k}, d_{k}\right)$ and $t \in\left[t_{k}, t_{k}+d_{k}\right]$, then $W_{i}(t)=1$. Otherwise, $W_{i}(t)=$ 0

$\boldsymbol{Y}(t)$ is the system output, i.e., the production count at the last machine $S_{M}$ in the system.

Denote $\boldsymbol{\theta}(t)=\left[\theta_{1}(t), \theta_{2}(t), \ldots, \theta_{M}(t)\right]^{\prime}$ to be the status of all machines at time $t$, where $\theta_{i}(t)$ represents the status of machine $S_{i}$, which is either up $\left(\theta_{i}(t)=1\right)$ or down $\left(\theta_{i}(t)=0\right)$. It is not hard to see that machine $S_{i}$ is up at time $t$ only if $u_{i}(t)=1$ and $W_{i}(t)=0$, i.e., $\theta_{i}(t)=u_{i}(t)\left(1-W_{i}(t)\right)$.

Based on the conservation of flow, the accumulated production counts within a time period $[0, t]$ between any two machines $S_{i}$ and $S_{j}, \forall i, j \in\{1, \ldots, M\}, i \neq j$, satisfy the following equation:

$$
X_{i}(t)-X_{j}(t)=\tau_{i j}(t)=\left\{\begin{array}{l}
\sum_{k=i+1}^{j} b_{k}(t)-\sum_{k=i+1}^{j} b_{k}(0), i<j \\
\sum_{k=j+1}^{i} b_{k}(0)-\sum_{k=j+1}^{i} b_{k}(t), i>j
\end{array}\right.
$$

Note that $\tau_{i j}(t)$ has an upper bound, which is the condition that all buffers between machines $S_{i}$ and $S_{j}$ are full (for $i<j$ ) or empty (for $i>j$ ). Let $\beta_{i j}$ denotes this boundary, then we have

$$
\beta_{i j}=\left\{\begin{array}{r}
\sum_{k=i+1}^{j} B_{k}-\sum_{k=i+1}^{j} b_{k}(0), i<j \\
\sum_{k=j+1}^{i} b_{k}(0), i>j
\end{array}\right.
$$

Therefore $X_{i}(t)-X_{j}(t)=\tau_{i j}(t)$ cannot exceed this boundary, i.e., $\tau_{i j}(t) \leq \beta_{i j}$. In case of $X_{i}(t)-$ $X_{j}(t)<\beta_{i j}$, machine $S_{i}$ is not starved or blocked by machine $S_{j}$. In case of $X_{i}(t)-X_{j}(t)=\beta_{i j}$, process at machine $S_{i}$ will be constrained by machine $S_{j}$, i.e., the processing speed of $S_{i}$ will be altered to that of $S_{j}$. Therefore, an operational machine either processes at its own rated speed (when it is neither blocked nor starved) or at the speed of other machines. Denote $\gamma_{i}(t)=\theta_{i}(t) / T_{i}=u_{i}(t)\left(1-W_{i}(t)\right) / T_{i}$, if $X_{i}(t)-$ $X_{j}(t)=\beta_{i j}$, then $\dot{X}_{i}(t)=\min \left\{\gamma_{i}(t), \gamma_{j}(t)\right\}$; if $X_{i}(t)-X_{j}(t)<\beta_{i j}$, then $\dot{X}_{i}(t)=\gamma_{i}(t)$. It can be summarized as 


$$
\dot{X}_{i}(t)=\min \left\{\frac{\zeta\left(\left(X_{i}(t)-X_{j}(t)\right)-\beta_{i j}, u_{j}(t)\left(1-W_{j}(t)\right)\right)}{T_{j}}, \frac{u_{i}(t)\left(1-W_{i}(t)\right)}{T_{i}}\right\}
$$

where $\zeta(u, v)$ is a segment function defined as $\zeta(u, v)=\left\{\begin{array}{r}+\infty, u<0 \\ v, u=0\end{array}\right.$.

Eq. (6) can be extended for all machines when comparing with machine $S_{i}$, then

$$
\dot{X}_{i}(t)=\min \left\{\begin{array}{c}
\frac{\zeta\left(\left(X_{i}(t)-X_{1}(t)\right)-\beta_{i 1}, u_{1}(t)\left(1-W_{1}(t)\right)\right)}{T_{1}}, \\
\frac{\zeta\left(\left(X_{i}(t)-X_{2}(t)\right)-\beta_{i 2}, u_{2}(t)\left(1-W_{2}(t)\right)\right)}{T_{2}}, \\
\vdots \\
\frac{u_{i}(t)\left(1-W_{i}(t)\right)}{T_{i}}, \\
\vdots \\
\frac{\zeta\left(\left(X_{i}(t)-X_{M}(t)\right)-\beta_{i M}, u_{M}(t)\left(1-W_{M}(t)\right)\right)}{T_{M}}
\end{array}\right\}=f_{i}(\boldsymbol{X}(t), \boldsymbol{U}(t), \boldsymbol{W}(t))
$$

To summarize all machines, the state space function of $\dot{\boldsymbol{X}}(t)$ is

$$
\begin{aligned}
& \dot{\boldsymbol{X}}(t)=\left[\begin{array}{c}
f_{1}(\boldsymbol{X}(t), \boldsymbol{U}(t), \boldsymbol{W}(t)) \\
\vdots \\
f_{M}(\boldsymbol{X}(t), \boldsymbol{U}(t), \boldsymbol{W}(t))
\end{array}\right]=\boldsymbol{F}(\boldsymbol{X}(t), \boldsymbol{U}(t), \boldsymbol{W}(t)) \\
& \boldsymbol{Y}(t)=X_{M}(t)=\left[\begin{array}{llll}
0 & \ldots & 0 & 1
\end{array}\right] \boldsymbol{X}(t)=\boldsymbol{H}(\boldsymbol{X}(t))
\end{aligned}
$$

Therefore, $\boldsymbol{F}(*)$ and $\boldsymbol{H}(*)$ are specified, and suppose probability distribution of the random variables in $\boldsymbol{W}(t)$ are also specified. The stochastic manufacturing model is then specified according to Definition 1 . In addition, the buffer level of a certain buffer $B_{i+1}$ at time $t$ can be easily evaluated as

$$
b_{i+1}(t)=X_{i}(t)-X_{i+1}(t)+b_{i+1}(0)
$$

Then the buffer states at time $t$, denoted as $\boldsymbol{B}(t)=\left[b_{2}(t), b_{3}(t), \ldots, b_{M}(t)\right]$, is obtained. The mathematical model of the serial production line system including buffer levels is then described by Eqs. (8)(10).

This system model is built upon sensor data. Given the initial buffer levels $b_{2}(0), \ldots, b_{M}(0)$ and sensor data of random disruption events $\boldsymbol{E}$, the detailed system performance at any given time can be iteratively evaluated which is different from steady state performance evaluation. Among the most important measures of the production system performance are energy saving opportunity, permanent production loss, and how each machine and disruption event contribute to the loss. This is discussed in the next section.

\section{Energy saving opportunity and production loss analysis}

In order to identify the energy saving opportunity and permanent production loss, it is necessary to understand how disruptions events affect the system. From our previous studies [13], [16], [36], it is discovered that not every disruption event leads to permanent production loss. To keep this paper selfcontained, we briefly summarize the related concepts without detailed proof. 
Definition 2: Energy saving opportunity window $\operatorname{ESO}_{m}\left(T_{d}\right)$ is the longest possible disruption time on machine $S_{m}$ at time $T_{d}$ that does not result in permanent production loss at the end-of-line machine [13], [16], [36], i.e.

$$
E S O_{m}\left(T_{d}\right)=\sup \left\{d \geq 0: s . t . \exists T^{*}(d), \int_{0}^{T} s_{M}(t) d t=\int_{0}^{T} \tilde{s}_{M}(t ; \vec{e}) d t, \forall T \geq T^{*}(d)\right\}
$$

where $\int_{0}^{T} \tilde{s}_{M}(t ; \vec{e}) d t$ and $\int_{0}^{T} S_{M}(t) d t$ are the production volume of the end-of-line machine $S_{M}$ at time $T$, with and without disruption event $\vec{e}_{i}=\left(m, T_{d}, d\right)$, respectively. $T^{*}(d)$ signifies the potential dependency of $T^{*}$ on $d$.

The definition of the energy saving opportunity window requires the production volume to recover as if no disruption event had occurred and only disruption event longer than the energy saving opportunity window causes permanent production loss at the end-of-line machine. This energy saving opportunity window can be utilized for energy savings without impacting the system production. For instance, machine $S_{m}$ can be forced to energy saving mode or shut down mode at time $T_{d}$ with a duration of $\operatorname{ESO}_{m}\left(T_{d}\right)$.

Furthermore, the permanent production loss due to an elongated disruption at a single machine is also permanent to all other machines in the line. If the duration of a disruption event $\vec{e}=(j, t, d)$ is greater than its corresponding energy saving opportunity window $\operatorname{ESO}_{j}(t)$, then for any machine $S_{i}$ in the line, there exists $T^{*} \geq t+d$, which may depend on the relative location of machine $S_{j}$ with respect to the slowest machine $S_{M^{*}}$, such that

$$
\int_{0}^{T} s_{j}\left(t^{\prime}\right) d t^{\prime}-\int_{0}^{T} s_{j}\left(t^{\prime}, \vec{e}\right) d t^{\prime}=\frac{d-E S O_{j}(t)}{T_{M^{*}}}, \forall T>T^{*}
$$

In other words, all machines in the transfer line permanently lose $\left(d-E S O_{j}(t)\right) / T_{M^{*}}$ units of production when the disruption event $\vec{e}=(j, t, d)$ lasts longer than the energy saving opportunity window $\operatorname{ESO}_{j}(t)$. Consequently, this loss of production at all machines can be interpreted as the production loss of the whole production line.

Proposition 1. [36] Given a realization of a production process subject to a sequence of disruption events $\boldsymbol{E}=\left[\vec{e}_{1}, \ldots, \vec{e}_{n}\right]$ and suppose $\max _{l=1, \ldots, n}\left\{t_{l}+d_{l}\right\}<T^{\prime}$, if the last slowest machine $S_{M^{*}}$ stops for a duration of $D$ during time $\left[0, T^{\prime}\right)$, then for the end-of-line machine $S_{M}, \exists T^{*} \geq T^{\prime}$, s.t.

$$
X_{M}(T)-X_{M}(T ; E)=D / T_{M^{*}}, \forall T>T^{*}
$$

where $X_{M}(T ; \boldsymbol{E})$ and $X_{M}(T)$ are the production of the end-of-line machine $S_{M}$ with and without disruption events $\boldsymbol{E}=\left[\vec{e}_{1}, \ldots, \vec{e}_{n}\right]$ during $[0, T)$.

From Proposition 1, we can conclude that any stoppage of the last slowest machine $S_{M^{*}}$ contributes to the permanent production loss of the whole line. Note that the stoppage includes not only the disruption events on machine $S_{M^{*}}$ due to failure, but also the temporary stoppage due to blockage or starvation.

Given the system model described by Eqs. (8)-(10), the states $\boldsymbol{X}(t)$ and $\boldsymbol{B}(t)$ need to be derived at any given time in order to evaluate the permanent production loss caused by each machine. However, for a stochastic production system, it is very challenging to predict the system states. Therefore, production loss analysis is discussed in two scenarios, namely, deterministic scenario and stochastic scenario. 


\subsection{Production loss diagnosis under deterministic scenario}

In the deterministic scenario, $\boldsymbol{W}(t)$ can be fully observed without uncertainty. Therefore, the states $\boldsymbol{X}(t)$ and $\boldsymbol{B}(t)$, i.e., the production counts for all machines and buffer levels for all buffers at any time during observation horizon $[0, T]$ can be iteratively calculated using Eqs. (8)-(10). Permanent production loss for the production line during a time period $[0, T]$ can be obtained from real production output $\boldsymbol{Y}(t)$ and the production count by the last slowest machine as:

$$
P L(T)=\frac{T}{T_{M^{*}}}-X_{M}(T)=\frac{T}{T_{M^{*}}}-\boldsymbol{Y}(T)
$$

where $P L(T)$ represents deterministic production loss during a time period $[0, T]$. Note that Eq. (14) is in agreement with Proposition 1. This production loss needs to be further attributed to each event and machine.

For a realized production process subject to a known disruption event $\vec{e}_{i}=\left(j, t_{i}, d_{i}\right)$ within $[0, T]$, we have $X_{j}(t)=X_{j}\left(t_{i}\right), \forall t \in\left[t_{i}, t_{i}+d_{i}\right]$, since machine $S_{j}$ stops from $t_{i}$. It is clear from Eq. (13) that every unit of permanent production loss can be related to the stoppage of the last slowest machine $S_{M^{*}}$. In the case when $\vec{e}_{i}$ dose not overlap with any other disruption event, the production loss due to $\vec{e}_{i}$ within $\left[t_{i}, t_{i}+d_{i}\right]$ is

$$
P L_{\vec{e}_{i}}\left[t_{i}, t_{i}+d_{i}\right]=\max \left\{0,\left(X_{M^{*}}\left(t_{i}\right)+d_{i} / T_{M^{*}}\right)-X_{j}\left(t_{i}\right)-\beta_{M^{*} j}\right\}
$$

where $P L_{\vec{e}_{i}}$ denotes the deterministic production loss due to $\vec{e}_{i}$ and $\beta_{M^{*} j}$ represents a special condition that the buffers between machine $S_{j}$ and $M^{*}$ become full $\left(M^{*}<j\right)$ or empty $\left(M^{*}>j\right)$ as

$$
\beta_{M^{*} j}=\left\{\begin{aligned}
\sum_{k=M^{*}+1}^{j}\left(B_{k}-b_{k}(0)\right), & M^{*}<j \\
0, & M^{*}=j \\
\sum_{k=j+1}^{M^{*}} b_{k}(0), & M^{*}>j
\end{aligned}\right.
$$

Eq. (15) indicates that the permanent production loss will start to accumulate after the buffers between machine $S_{j}$ and the last slowest machine $S_{M^{*}}$ become full $\left(M^{*}<j\right)$ or empty $\left(M^{*}>j\right)$. If, however, $\vec{e}_{i}$ overlaps with other disruption events then the permanent production loss in the overlapping periods can be attributed to the involved disruption events based on any reasonable principles. For example, the permanent production loss can account to the disruption event which happens the first or can be equally shared among the corresponding events. Indeed, each attribution argument has its own appeal, but the difference is purely technical in nature and there is no significant advantage of one over another. To eliminate ambiguity and without loss of generality, production loss during the overlapping period will be equally shared among the corresponding events in this paper.

Based on $P L_{\vec{e}_{i}}$, the production loss can be further attributed to each machine. For a realized production process, there exists a sequence of disruption events $\vec{e}_{j, 1}, \ldots \vec{e}_{j, n}$ on machine $S_{j}$ during a time period $[0, T]$. The deterministic permanent production loss caused by machine $S_{j}$ within $[0, T]$ is denoted as $P L_{j}(T)$, which can be derived by

$$
P L_{j}(T)=\sum_{q=1}^{n} P L_{\vec{e}_{j, q}}
$$




\subsection{Production loss prognosis under stochastic scenario}

In a stochastic scenario, suppose the information of the system states at time $t$ is observable, the challenge is to predict the potential behavior of the production system in face of random disruption events. Since production systems are complicated stochastic dynamic systems, for a given set of $\boldsymbol{X}(t), \boldsymbol{B}(t)$ and $\boldsymbol{\theta}(t)$ at time $t$, it is unlikely to derive a general complete prediction about the system performance, such as $\boldsymbol{X}\left(t^{\prime}\right)$, $\boldsymbol{B}\left(t^{\prime}\right)$, and potential production loss for $\forall t^{\prime}>t$. Therefore, to make the computation and production control tractable, a "look ahead window" $\Delta t$ is selected such that $\Delta t \ll M T B F_{i}, \forall i=1, \ldots, M$. Under this condition, the system performance can be predicted by evaluating potential production loss due to single disruption event during "look ahead window" period $[t, t+\Delta t]$. The prediction will continuously update as time $t$ moving forward. It is noted that the probability distribution of potential production loss at time $t+\Delta t$ only depends on the system and buffer status in the present time moment $t$, i.e., Markov property is applied. Denote $\vec{e}=\left(j, t^{*}, \sigma\right), t+\Delta t>t^{*} \geq t$, as the single disruption event during $[t, t+\Delta t)$. Therefore, $j, t^{*}$, and $\sigma$ are the disruption machine number, start time, and duration of the disruption event, accordingly. Let $p\left(j, t^{*}, \sigma\right)$ be the joint probability density function of $j, t^{*}$, and $\sigma . P P L_{j}\left(t, t^{*}, \sigma\right)$ represents the future potential production loss attributed to machine $S_{j}$ within the time period $[t, t+\Delta t)$, due to the disruption event $\vec{e}=\left(j, t^{*}, \sigma\right)$ on machine $S_{j}$. For a production process subjected to $\vec{e}=\left(j, t^{*}, \sigma\right)$, we have $X_{j}\left(t_{1}\right)=$ $X_{j}\left(t^{*}\right), \forall t_{1} \in\left[t^{*}, t^{*}+\sigma\right]$, since machine $S_{j}$ stops from time $t^{*}$. Noted that if $\vec{e}=\left(j, t^{*}, \sigma\right)$ starts within $[t, t+\Delta t)$ and ends after $t+\Delta t$, i.e., $t^{*} \in[t, t+\Delta t)$ and $\sigma>t+\Delta t-t^{*}$, its impact on the system during $[t, t+\Delta t)$ is equivalent to the disruption event starts at $t^{*}$ but ends at $t+\Delta t$, i.e., $\vec{e}=\left(j, t^{*}, t+\Delta t-t^{*}\right)$. Similar to the analysis in deriving Eq. (15), we have

$$
P P L_{j}\left(t, t^{*}, \sigma\right)=\left\{\begin{array}{r}
\max \left\{0,\left(X_{M^{*}}\left(t^{*}\right)+\frac{\sigma}{T_{M^{*}}}\right)-X_{j}\left(t^{*}\right)-\beta_{M^{*} j}\right\}, \\
\quad \text { or } t^{*} \in[t, t+\Delta t) \text { and } \sigma<t+\Delta t-t^{*} \\
\max \left\{0,\left(X_{M^{*}}\left(t^{*}\right)+\frac{t+\Delta t-t^{*}}{T_{M^{*}}}\right)-X_{j}\left(t^{*}\right)-\beta_{M^{*} j}\right\}, \\
\quad \text { for } t^{*} \in[t, t+\Delta t) \text { and } \sigma \geq t+\Delta t-t^{*}
\end{array}\right.
$$

Eq. (18) means that if a future disruption event makes the buffers between machine $S_{j}$ and the last slowest machine $S_{M^{*}}$ full $\left(M^{*}<j\right)$ or empty $\left(M^{*}>j\right)$, then potential production loss will start to occur. According to Eq. (4),

$$
X_{M^{*}}\left(t^{*}\right)-X_{j}\left(t^{*}\right)=\tau_{M^{*} j}\left(t^{*}\right)=\left\{\begin{aligned}
\sum_{k=M^{*}+1}^{j} b_{k}\left(t^{*}\right)-\sum_{k=M^{*}+1}^{j} b_{k}(0), & M^{*}<j \\
0, & M^{*}=j \\
\sum_{k=j+1}^{M^{*}} b_{k}(0)-\sum_{k=j+1}^{M^{*}} b_{k}\left(t^{*}\right), & M^{*}>j
\end{aligned}\right.
$$

Now, inserting Eq. (19) into Eq. (18) yields

$$
P P L_{j}\left(t, t^{*}, \sigma\right)=P P L_{j}\left(t, t^{*}, \sigma, \tau_{M^{*} j}\left(t^{*}\right)\right)=\left\{\begin{aligned}
& \max \left\{0, \frac{\sigma}{T_{M^{*}}}-\beta_{M^{*} j}+\tau_{M^{*} j}\left(t^{*}\right)\right\}, \\
& \text { for } t^{*} \in[t, t+\Delta t] \text { and } \sigma<t+\Delta t-t^{*} \\
& \max \left\{0, \frac{t+\Delta t-t^{*}}{T_{M^{*}}}-\beta_{M^{*} j}+\tau_{M^{*} j}\left(t^{*}\right)\right\}, \\
& \quad \text { for } t^{*} \in[t, t+\Delta t] \text { and } \sigma \geq t+\Delta t-t^{*}
\end{aligned}\right.
$$


With an abuse of notation, we use $P P L_{j}\left(t, t^{*}, \sigma, \tau_{M^{*} j}\left(t^{*}\right)\right)$ to further represent the dependencies of potential production loss on $\tau_{M^{*} j}\left(t^{*}\right)$. Then the expected potential production loss attributed to machine $S_{j}$, caused by the single disruption event within $[t, t+\Delta t)$ can be evaluated as

$$
E\left[P P L_{j}\left(t, t^{*}, \sigma, \tau_{M^{*} j}\left(t^{*}\right)\right)\right]=\int_{t}^{t+\Delta t} \int_{0}^{\infty} p\left(j, t^{*}, \sigma\right) P P L_{j}\left(t, t^{*}, \sigma, \tau_{M^{*} j}\left(t^{*}\right)\right) d \sigma d t^{*}
$$

It is clear that $\tau_{M^{*} j}\left(t^{*}\right)$ needs to be estimated in order to predict $E\left[P P L_{j}\left(t, t^{*}, \sigma, \tau_{M^{*} j}\left(t^{*}\right)\right)\right]$. However, the status of machines, $\boldsymbol{\theta}\left(t_{1}\right), \forall t_{1} \in\left(t, t^{*}\right]$, are not observable at time $t$. Since there is no random disruptions during $\left(t, t^{*}\right], \tau_{M^{*} j}\left(t^{*}\right)$ can be evaluated based on Eq. (19). Furthermore, there exists an upper bound for the expected potential production loss, which is described in the following proposition.

Proposition 2. For a realization of a production process at time $t$, the first disruption event during $[t, t+\Delta t)$ happens at machine $S_{j}$ as $\vec{e}=\left(j, t^{*}, \sigma\right), t+\Delta t>t^{*} \geq t$. Assume all machines are "on" during $\left[t, t^{*}\right]$, then the upper bound of the expected potential production loss due to a disruption event at machine $S_{j}$ is $E\left[P P L_{j}\left(t, t^{*}, \sigma, \tau_{M^{*} j}(t)\right)\right]$, i.e., $E\left[P P L_{j}\left(t, t^{*}, \sigma, \tau_{M^{*} j}\left(t^{*}\right)\right)\right] \leq E\left[P P L_{j}\left(t, t^{*}, \sigma, \tau_{M^{*} j}(t)\right)\right]$.

Proof. Since $S_{M^{*}}$ is the slowest machine, $\forall t_{1} \in\left[t, t^{*}\right], \dot{X}_{j}\left(t_{1}\right) \geq \dot{X}_{M^{*}}\left(t_{1}\right)$, i.e., $\dot{X}_{j}\left(t_{1}\right)-\dot{X}_{M^{*}}\left(t_{1}\right)=$ $\dot{\tau}_{M^{*} j}\left(t_{1}\right) \geq 0$. Therefore, $\tau_{M^{*} j}\left(t^{*}\right) \geq \tau_{M^{*} j}\left(t_{1}\right) \geq \tau_{M^{*} j}(t)$. Plugging this relationship into Eq. (20), we have $P P L_{j}\left(t, t^{*}, \sigma, \tau_{M^{*} j}\left(t^{*}\right)\right) \geq P P L_{j}\left(t, t^{*}, \sigma, \tau_{M^{*} j}(t)\right)$. Note that the probability density function $p\left(j, t^{*}, \sigma\right) \geq 0$, then

$$
\begin{aligned}
E\left[P P L_{j}\left(t, t^{*}, \sigma, \tau_{M^{*} j}\left(t^{*}\right)\right)\right]=\int_{t}^{t+\Delta t} \int_{0}^{\infty} p\left(j, t^{*}, \sigma\right) P P L_{j}\left(t, t^{*}, \sigma, \tau_{M^{*} j}\left(t^{*}\right)\right) d \sigma d t^{*} \\
\leq \int_{t}^{t+\Delta t} \int_{0}^{\infty} p\left(j, t^{*}, \sigma\right) P P L_{j}\left(t, t^{*}, \sigma, \tau_{M^{*} j}(t)\right) d \sigma d t^{*}=E\left[P P L_{j}\left(t, t^{*}, \sigma, \tau_{M^{*} j}(t)\right)\right]
\end{aligned}
$$

\section{End of proof.}

Since $\vec{e}=\left(j, t^{*}, \sigma\right)$ is the first only disruption event within $[t, t+\Delta t)$, there is no other disruption during $\left[t, t^{*}\right] . \tau_{M^{*} j}\left(t^{*}\right)$ can be easily obtained to estimate $E\left[P P L_{j}\left(t^{*}, \sigma, \tau_{M^{*} j}\left(t^{*}\right)\right)\right]$. To simplify the calculation, one can also use the upper bound $E\left[P P L_{j}\left(t^{*}, \sigma, \tau_{M^{*} j}(t)\right)\right]$ to approximate $E\left[P P L_{j}\left(t^{*}, \sigma, \tau_{M^{*} j}\left(t^{*}\right)\right)\right]$ for realtime control.

Note that $p\left(j, t^{*}, \sigma\right)$ can be any probability density function. As an illustration, we specify that for any machine $S_{i}$ in the line, its time between failure $\left(T B F_{i}\right)$ and time to repair $\left(T T R_{i}\right)$ follow exponential distribution $\exp \left(\lambda_{i}\right)$ and $\exp \left(\mu_{i}\right)$, accordingly. The expected potential production loss can be explicitly presented. Since $T B F_{i}$ and $T T R_{i}$ are independent of each other, i.e., $\sigma$ is independent of $t^{*}$, then

$$
p\left(j, t^{*}, \sigma\right)=p\left(j, t^{*}\right) p_{j}(\sigma)
$$

where $p\left(j, t^{*}\right)$ is the joint probability density function of $j$ and $t^{*}$, which follows the probability distribution of the first arrival time of $M$ independent exponential processes, i.e.,

$$
p\left(j, t^{*}\right)=\lambda_{j} e^{-\left(\lambda_{1}+\lambda_{2}+\cdots+\lambda_{M}\right)\left(t^{*}-t\right)}
$$


and $p_{j}(\sigma)$ is the conditional probability density function of $\sigma$ given $j$, i.e., the duration of the disruption event on machine $S_{j}$, which is

$$
p_{j}(\sigma)=\mu_{j} e^{-\mu_{j} \sigma}
$$

Substitute Eqs. (22) and (23) into Eq. (24) yields

$$
p\left(j, t^{*}, \sigma\right)=\lambda_{j} \mu_{j} e^{-\left(\lambda_{1}+\lambda_{2}+\cdots+\lambda_{M}\right)\left(t^{*}-t\right)} e^{-\mu_{j} \sigma}
$$

Then according to Eq. (21), the expected potential production loss $E\left[P P L_{j}\left(t, t^{*}, \sigma, \tau_{M^{*} j}(t)\right)\right]$ is

$$
E\left[P P L_{j}\left(t, t^{*}, \sigma, \tau_{M^{*} j}(t)\right)\right]=\int_{t}^{t+\Delta t} \int_{0}^{\infty} \Psi d \sigma d t^{*}
$$

where $\Psi=\lambda_{j} \mu_{j} e^{-\left(\lambda_{1}+\lambda_{2}+\cdots+\lambda_{M}\right)\left(t^{*}-t\right)-\mu_{j} \sigma} P P L_{j}\left(t, t^{*}, \sigma, \tau_{M^{*} j}(t)\right)$.

If there happens to be a disruption event at the start of the "look ahead window", i.e., $t^{*}=t$, then Eqn. (22) becomes $p\left(j, t^{*}, \sigma\right)=p(j, \sigma)=p_{j}(\sigma)$ since the time of the disruption event is known, and hence Eqn. (25) is simplified to be Eqn. (24). Then potential production loss $E\left[P P L_{j}\left(t, t^{*}, \sigma, \tau_{M^{*} j}(t)\right)\right]$ is also simplified accordingly.

\section{Distributed feedback control law}

It is well known that for complex production system, there is a lack of general complete prediction for system dynamic status at any future time. Although long-term steady-state performance measurements may be estimated, they cannot be used for optimal real-time control. Hence, it is extremely difficult, if not possible, to find an optimal control policy for a global large time period $[0, T]$. However, using system diagnostic and prognostic information acquired in Section 5, instead of optimal control policy, a distributed feedback control policy can be designed in real time fashion for individual machine to effectively improve the system profit and overall energy efficiency. Fig. 2 is a schematic of control input determined by a control cost function. When time $t$ moves forward on a global time period $[0, T]$, the control inputs will be continuously updated at each local time instant based on a control cost function that considers both current system performance and potential system performance in "look ahead window". Note that the control cost function is also related to real-time system status, and thus may change over time due to system status variation. For example, in Fig. 2, the control cost function at time $t_{1}$ and $t_{1}+1$ are different. It is the first ever production control algorithm by utilizing rigorous control theory in stochastic manufacturing systems.

\section{Insert Fig. 2 about here}

\subsection{Control framework}

The control inputs $\boldsymbol{U}(t)$ are decided by the current states of the production system and we have $\boldsymbol{U}(t)=$ $\boldsymbol{G}(\boldsymbol{X}(t), \boldsymbol{\theta}(t))$. In this paper, the control inputs are switching each machine on or off. The feedback policy $\boldsymbol{G}(*)$ depends on the current system states $\boldsymbol{X}(t)$ and $\boldsymbol{\theta}(t)$. Therefore, $\boldsymbol{G}(*)$ is a Markov policy. The structure of the control framework is shown in Fig. 3. 


\section{Insert Fig. 3 about here}

Different policies $\boldsymbol{G}(*)$ will lead to different probability distribution and system states. The corresponding cost of a Markov policy is evaluated by a real-time control cost function. In this paper, the purpose of the control is to perform hold/release operations on each machine to minimize the real-time control cost. Due to the complexity of general production system, it takes extraordinary computational effort to evaluate the overall cost function of the entire production system. For example, for a 20 machine production system, there are $2^{20}=1048576$ options for each machine turning on/off and it will be a tremendous computational effort for the controller to evaluate the control cost function of all those options in real-time. To avoid intense calculation, a distributed control policy is created and a cost function is established at individual machine level to determine the control input for each machine to be on or off. Hence, the workload for the controller will then be enormously reduced. Still taking the 20 machine production system as an example, the number of options is decreased to $2 \times 20=40$.

The distributed feedback controller is the integration of a local control policy and a collaborated control policy. The local feedback policy considers only individual machine $S_{j}$ 's impact to the system. The collaborated control is achieved by considering whether machine $S_{j}$ is in the risk of constraining the operation of its neighborhood machines. In this way, we only need to evaluate the cost of two control options (on and off) for each machine $S_{j}$ and locally determine the optimal policy.

The goal of the distributed control is to minimize the real-time expense of the system (e.g., energy expense) without causing extra production loss or bringing in the potential production loss to an unacceptable high level, as well as avoiding frequent change of machines' status. Therefore, the real-time control cost function for an arbitrary machine $S_{j}$ is formulated as:

$$
C_{j}(t)=C_{j, l o c}(t)+C_{j, c o l}(t)
$$

where $C_{j, l o c}(t)$ stands for real-time local control cost at machine $S_{j}$ and $C_{j, c o l}(t)$ stands for real-time collaboration request cost at machine $S_{j}$ due to collaborated control.

(1) Real-time local control cost $C_{j, l o c}(t)$

We formulate the real-time local control cost $C_{j, l o c}(t)$ as

$$
C_{j, l o c}(t)=C_{j, E}(t)+C_{j, P L}(t)+C_{j, P P L}(t)+C_{j, S w}(t)
$$

where $C_{j, E}(t)$ is the real-time energy cost of machine $S_{j}, C_{j, P L}(t)$ denotes the cost of real-time production loss attributed to machine $S_{j}, C_{j, P P L}(t)$ represents the cost of potential production loss attributed to machine $S_{j}$, and $C_{j, s w}(t)$ is the status switching cost of machine $S_{j}$ at time $t$.

$C_{j, E}(t)$ is evaluated by the cost of energy used by machine $S_{j}$ within a unit time in its current consumption rate.

$$
C_{j, E}(t)=c_{e} K_{j} I_{t} \theta_{j}(t)
$$

where $c_{e}$ is the electricity rate, $K_{j}$ is the power consumption rate of machine $S_{j}$, and $I_{t}$ is the length of a unit time.

At time $t$, machine $S_{j}$ causes production loss when it blocks or starves machine $S_{M^{*}}$, which is equivalent to $\theta_{j}(t)=0$ and $\tau_{M^{*} j}(t)=\beta_{M^{*} j}$. Referring to Eq. (17), we can evaluate $\dot{P} L_{j}(t)$, which is the production loss speed caused by machine $S_{j}$ at the time $t . C_{j, P L}(t)$ is then calculated as the cost of production loss due to machine $S_{j}$ within a unit time based on $\dot{P} L_{j}(t)$ : 


$$
C_{j, P L}(t)=c_{p} I_{t} \dot{P L_{j}}(t)
$$

where $c_{p}$ is the profit per part produced.

The potential production loss as discussed in the previous section needs to be considered in the control cost function, since real-time decision directly impacts potential production loss and system overall performance in the near future. There is no systematic way on how to account this future potential production loss. However, a discount ratio can be selected based on current production status and prioritization preference to include the future potential impact. Therefore, $C_{j, P P L}(t)$ is represented as

$$
C_{j, P P L}(t)=r c_{p}\left(1-\theta_{j}(t)\right) E\left[P P L_{j}\left(t, t^{*}, \sigma, \tau_{M^{*} j}(t)\right)\right]
$$

where $r$ is a discount ratio. When machine $S_{j}$ is on at time $t$, this discounted cost is removed since machine $S_{j}$ is in its best effort to avoid potential production loss.

There is a penalty cost by switching machines on and off. This cost accounts for the potential damage to the machine and extra energy cost when ramp up a machine from off to on. The switching cost of machine $S_{j}$ at time $t$ is valued by a function $w_{j}\left(\varphi_{j}(t)\right)$, where $\varphi_{j}(t)$ denotes the length of the interval machine $S_{j}$ stays in its current status $\theta_{j}(t)$. Therefore, $C_{j, s w}(t)$ is

$$
C_{j, s w}(t)=\left\{\begin{array}{rr}
w_{j}\left(\varphi_{j}(t)\right), & \theta_{j}(t) \neq \theta_{j}\left(t^{-}\right) \\
0, & \theta_{j}(t)=\theta_{j}\left(t^{-}\right)
\end{array}\right.
$$

(2) Real-time collaboration request $\operatorname{cost} C_{j, \text { col }}(t)$

For a machine $S_{j}$, when $j<M^{*}$, the neighborhood machine will be the immediate downstream machine of machine $S_{j}$, i.e., machine $S_{j+1}$, since machine $S_{j+1}$ has a smaller energy saving opportunity window which leads to a faster permanent production loss and may require $S_{j}$ to operate to avoid starving machine $S_{M^{*}}$. When $j>M^{*}$, the neighborhood machine will be the immediate upstream machine of machine $S_{j}$, i.e., machine $S_{j-1}$, since machine $S_{j-1}$ has a smaller energy saving opportunity window which leads to a faster permanent production loss and may require machine $S_{j}$ to operate to avoid blocking machine $S_{M^{*}}$. The cost of the request related to the production loss and potential production loss of the neighborhood machine $S_{j^{\prime}}$ can be evaluated as $r_{2}\left(C_{j^{\prime}, P L}(t)+C_{j^{\prime}, P P L}(t)\right)$, where $r_{2}$ is treated as collaboration ratio. There is also no systematic way to account this collaboration cost. Therefore, the collaboration ratio can be selected depending on real situation and user preference. Machine $S_{j}$ is responsible for production loss or potential production loss caused by machine $S_{j^{\prime}}$ under the condition that machine $S_{j}$ is jeopardizing the neighborhood machine $S_{j^{\prime}}$ to become starved, i.e., $b_{j^{\prime}}(t) \rightarrow 0$ (for $j<M^{*}$ ), or blocked, i.e., $b_{j^{\prime}+1}(t) \rightarrow B_{j^{\prime}+1}$ (for $j>M^{*}$ ). Therefore, the collaboration request cost at machine $S_{j}, C_{j, c o l}(t)$, is calculated by

$$
C_{j, \text { col }}(t)=\left\{\begin{array}{c}
0, \quad \text { if } \theta_{j}(t)=1 \\
r_{2} Z\left(b_{j^{\prime}}(t)\right)\left(C_{j^{\prime}, P L}(t)+C_{j^{\prime}, P P L}(t)\right), \\
\quad \text { if } \theta_{j}(t)=0 \text { and } j<M^{*} \\
r_{2} Z\left(B_{j^{\prime}+1}-b_{j^{\prime}+1}(t)\right)\left(C_{j^{\prime}, P L}(t)+C_{j^{\prime}, P P L}(t)\right), \\
\text { if } \theta_{j}(t)=0 \text { and } j>M^{*}
\end{array}\right.
$$


where $z(x)$ is a segment function with $z(x)=\left\{\begin{array}{l}0, x \geq \varepsilon_{0} \\ 1, x<\varepsilon_{0}\end{array}\right.$, and $\varepsilon_{0}$ is a selected threshold buffer value to indicate if machine $S_{j}$ is jeopardizing the neighborhood machine $S_{j^{\prime}}$.

Noted that the slowest machine $S_{M^{*}}$ has the smallest energy saving opportunity window $\left(E S O_{M^{*}}(t)=0\right)$. Based on the analysis of neighborhood machine in Section 6.1, $C_{M^{*}, \text { col }}(t)=0$ and $C_{M^{*}}(t)=C_{M^{*}, l o c}(t)$.

\subsection{Control law}

With the value of the cost function $C_{j}(t)$, we need to determine the optimal control input $u_{j}(t)$, i.e., turning the machine on or off, that minimizes $C_{j}(t)$. However, it is observed that $\forall j \neq M^{*}$, the collaboration request cost $C_{j, \text { col }}(t)$ as shown in Eq. (36) is also related to the status of the neighborhood machine, $\theta_{j^{\prime}}(t)=$ $\left(1-W_{j^{\prime}}(t)\right) u_{j^{\prime}}(t)$. Therefore, given $\boldsymbol{X}(t)$ and $\boldsymbol{W}(t), \forall j \neq M^{*}, C_{j}(t)=C_{j, l o c}(t)+C_{j, c o l}(t)$ depends on both $u_{j}(t)$ and $u_{j^{\prime}}(t)$. To find $u_{j}(t)=\arg \min _{u_{j}(t)} C_{j}(t)$, we need to know $u_{j^{\prime}}(t)$ in advance. Therefore, we start from the slowest machine $S_{M^{*}}$ since $C_{M^{*}}(t)=C_{M^{*}, l o c}(t)$. According to Eqs. (32)-(35), given $\boldsymbol{X}(t)$ and $\boldsymbol{W}(t)$, $C_{M^{*}}(t)$ is uniquely determined by $u_{M^{*}}(t)$ and thereby we can find $u_{M^{*}}(t)=\arg \min _{u_{M^{*}}(t)} C_{M^{*}}(t)$. The following pseudo procedures are used to describe how to determine the control action $u_{j}(t)$ for each machine.

\section{Procedure 1.1}

1. Given $\boldsymbol{X}(t)$ and $\boldsymbol{W}(t)$, initially set $j=M^{*}$ and find $u_{M^{*}}(t)=\arg \min _{u_{M^{*}}(t)} C_{M^{*}}(t)$;

2. Set machine number $j=j-1$, for which $j^{\prime}$ is the machine number in the previous step and $u_{j^{\prime}}(t)$ has been derived. Find $u_{j}(t)=\arg \min _{u_{j}(t)} C_{j}(t)$;

3. Repeat step 2 until $u_{j}(t)$ is solved for all upstream machines of machine $S_{M^{*}}$.

\section{Procedure 1.2}

1. Given $\boldsymbol{X}(t)$ and $\boldsymbol{W}(t)$, initially set $j=M^{*}$ and find $u_{M^{*}}(t)=\arg \min _{u_{M^{*}}(t)} C_{M^{*}}(t)$;

2. Set machine number $j=j+1$, for which $j^{\prime}$ is the machine number in the previous step and $u_{j^{\prime}}(t)$ has been derived. Find $u_{j}(t)=\arg \min _{u_{j}(t)} C_{j}(t)$;

3. Repeat step 2 until $u_{j}(t)$ is solved for all downstream machines of machine $S_{M^{*}}$.

Therefore, based on Procedure 1.1 and 1.2, we can derive $u_{j}(t)$ for all machines once $\boldsymbol{X}(t)$ and $\boldsymbol{W}(t)$ are given from sensor data. Hence, the corresponding feedback policy $\boldsymbol{G}(t)$ can be written as

$$
\boldsymbol{U}(t)=\boldsymbol{G}(\boldsymbol{X}(t), \boldsymbol{W}(t))
$$

Remark 1: The proposed control policy can be applied to any multi-stage production systems such as battery production lines, automotive manufacturing systems (both powertrain production and general assembly), etc. In these production systems, serial production lines are the basic structure and driving horse, and other layout can be simplified or aggregated to such structure [10].

\section{Energy economic analysis}

The proposed real-time distributed feedback control policy in Section 6 is established to improve system real-time profit and efficiency. To evaluate the effectiveness of the proposed control policy, we need to study 
the energy economics of the manufacturing system. First, the system profit within the time period $[0, T)$ is analyzed:

$$
\begin{aligned}
& \text { Profit }=\text { Total Revenue }- \text { Total Cost } \\
& \qquad \begin{array}{l}
=\text { Total Revenue }- \text { Total Energy Cost }- \text { Total Inventory Cost } \\
=(P C)\left(c_{p}\right)-C E-C I V
\end{array}
\end{aligned}
$$

where $P C$ is the system production count, $C E$ is the total cost of energy used by the production line, and $C I V$ is the total system inventory cost.

To find the total cost of energy consumption, i.e., $C E$, we analyze how each machine operates in $[0, T)$ :

$$
T=T_{o n, j}+T_{o f f, j}
$$

where $T_{o n, j}$ is the time machine $S_{j}$ is up and $T_{o f f, j}$ is the time machine $S_{j}$ is off.

The cost of energy consumption in $[0, T)$ is represented by

$$
C E=c_{e} \sum_{j=1}^{M} K_{j} T_{o n, j}(\$)
$$

The total inventory level of the production line at time $t$, denoted as $I L(t)$, is evaluated as

$$
I L(t)=\sum_{n=2}^{M} b_{n}(t)
$$

The total inventory cost in $[0, T)$ is then calculated as

$$
C I V=c_{i} \int_{0}^{T} I L(t) d t(\$)
$$

where $c_{i}$ is the inventory cost per part per day.

Inserting Eqs. (39)-(41) into Eq. (38) provides

$$
\text { Profit }=(P C)\left(c_{p}\right)-c_{e} \sum_{j=1}^{M} K_{j} T_{o n, j}-c_{i} \int_{0}^{T} I L(t) d t(\$)
$$

\section{Case study}

A case study is presented to illustrate the effectiveness of the real-time distributed feedback control law based on the stochastic production model and prediction. A production line as shown in Fig. 4 is used for the case study. It is a simplified version of a real automotive powertrain production line. The system parameters are shown in Table 1 and 2, which are recorded from the real production line and the data is mocked for confidential consideration. The $T B F_{i}$ and $T T R_{i}$ are assumed to be exponentially distributed based on the observed production data. The profit per part produced is assumed to be $c_{p}=\$ 300 /$ part. The electricity rate is assumed to be $c_{e}=\$ 0.2 / \mathrm{kWh}$. The inventory cost is assumed to be $c_{i}=\$ 50 /$ (part $*$ day). In this case study, discount ratio for potential production loss is selected as $r=0.5$, collaboration ratio is selected as 
$r_{2}=0.5$, and the length of a unit time is selected as $I_{t}=1 \mathrm{~min}$. The switching cost functions $w_{j}\left(\varphi_{j}(t)\right)($ the unit of $\varphi_{j}(t)$ is $\min$ ) for the machines are assumed to be simple linear functions as:

$$
\begin{aligned}
& w_{1}\left(\varphi_{1}(t)\right)=\max \left\{0,12-9 \varphi_{1}(t)\right\} \\
& w_{2}\left(\varphi_{2}(t)\right)=\max \left\{0,15-20 \varphi_{2}(t)\right\} \\
& w_{3}\left(\varphi_{3}(t)\right)=\max \left\{0,10-10 \varphi_{3}(t)\right\} \\
& w_{4}\left(\varphi_{4}(t)\right)=\max \left\{0,12-15 \varphi_{4}(t)\right\} \\
& w_{5}\left(\varphi_{5}(t)\right)=\max \left\{0,21-28 \varphi_{5}(t)\right\} \\
& w_{6}\left(\varphi_{6}(t)\right)=\max \left\{0,16-12 \varphi_{6}(t)\right\}
\end{aligned}
$$

\section{Insert Fig. 4 about here}

\section{Insert Table 1 about here}

Insert Table 2 about here

\section{Insert Table 3 about here}

\section{Insert Table 4 about here}

\section{Insert Table 5 about here}

\section{Insert Fig. 5 about here}

The simulation duration is three weeks, i.e., $30240 \mathrm{~min}$. Two scenarios are compared using simulation: 1) baseline scenario with no control of the production line; 2) controlled scenario with the proposed control law applying on the production line. The results, i.e., production count (PC), total cost of energy used by the machines (CE), overall profit (PF), and the mean value of total inventory level (MIL) can be seen in Table 3 with 95\% confidence interval (CI) included. CE and PF can be calculated by Eqs. (39) and (42), accordingly.

The production line performance improvements are demonstrated in Table 4. It is clear that the overall profit of the production system is well increased and the total cost of energy used by the machines is significantly reduced. The mean value of inventory level is also notably lowered which further reduces the overall manufacturing cost. It is worth noting that there is a minor throughput impact by applying the control, which does not conflict with the control goal. The control cost function focuses on overall profit and energy efficiency, which needs tradeoff among throughput and other cost factors.

If the value of profit per part produced is increased from $\$ 300 /$ part to $\$ 600 /$ part, the throughput impact should be lowered automatically through the distributed real-time control. Table 5 and 6 illustrate the corresponding results, and Fig. 5 shows the comparison between production control system performance with $c_{p}=\$ 300 /$ part and $c_{p}=\$ 600 /$ part . Careful examination of the results in Tables 3-6 and Fig. 5 suggests that by increasing $c_{p}$ from $\$ 300 /$ part to $\$ 600 /$ part: i) the throughput impact is reduced (from $2.7 \%$ to 
$1.2 \%$ ), and ii) the energy cost reduction (from $27.0 \%$ to $25.1 \%$ ) will be lowered through the distributed realtime control.

Remark 2: The performance of the proposed control policy is closely related to the value of $c_{p}$. Based on the cost function as described by Eqs. (30)-(36), the cost of the permanent production loss will be higher if $c_{p}$ is increased. As a result of the tradeoff between throughput cost and other cost factors, the control policy will automatically lower the throughput impact. It is also noted that the discount ratio for potential production loss and the collaboration ratio will also impact the final control result by changing the tradeoff between different cost factors. Depending on real scenario at the time moment, such ratios can be compared and adjusted based on users' preferences.

\section{Conclusion and future work}

In this paper, a mathematical model is established using the sensor data, where production counts are the state variables and disruption events are the stochastic input. The potential system energy saving opportunities can be evaluated based on energy saving opportunity window concept. The future potential production loss caused by each machine with respect to the current system states is estimated to evaluate the system response in face of random disruption events.

In addition, with the analytical results from the mathematical model and potential production loss estimation, a real-time distributed feedback control policy based on local and collaborate cost function is designed for each individual machine. This distributed feedback control policy can significantly reduce the energy consumption with minor throughput impact and increase the overall profit and energy efficiency for the production system.

In the future, we plan to estimate the potential production loss under the influence of multiple disruption events, which will release the assumptions on $T B F_{i}$ and $T T R_{i}$. Control parameters will be furthered studied for the proposed control policy to achieve higher profit and energy efficiency. Optimal control policy will be explored to maximize the overall profit over a long time period.

\section{Acknowledgements}

This work was supported by the U.S. National Science Foundation (NSF) Grant No. CMMI 1351160.

\section{References}

[1] Advanced Manufacturing Partnership 2.0. Annex 1: Transformative manufacturing technology: manufacturing technology area 1advanced sensing, control, and platforms for manufacturing https://www.whitehouse.gov/sites/default/files/microsites/ostp/PCAST/amp2.0-annex1-10-manufacturingtechnologyareas.pdf.

[2] Towill D R, Evans G N, Cheema P. Analysis and design of an adaptive minimum reasonable inventory control system. Prod Plan Control 1997; 8(6): 545-57. DOI: 10.1080/095372897234885.

[3] Wiendahl H P, Breithaupt J W. Automatic production control applying control theory. Int J Prod Econ 2000; 63(1): 33-46. doi:10.1016/S0925-5273(98)00253-9.

[4] Duffie N, Falu I. Control-theoretic analysis of a closed-loop PPC system. CIRP Ann Manuf Techno 2002; 51(1): 379-82. doi:10.1016/S-0007-8506(07)61541-8.

[5] Ratering A M, Duffie N A. Design and analysis of a closed-loop single workstation PPC system. CIRP Ann Manuf Techno 2003; 52(1): 355-58. doi:10.1016/S0007-8506(07)60600-3.

[6] Sethi S P, Yan H, Zhang H, Zhang Q. Optimal and hierarchical controls in dynamic stochastic manufacturing systems: a survey. Manuf Serv Oper Manag 2002; 4(2): 133-70. http://dx.doi.org/10.1287/msom.4.2.-133.281.

[7] Colledani M, Tolio T. Integrated quality, production logistics and maintenance analysis of multi-stage asynchronous manufacturing systems with degrading machines. CIRP Ann Manuf Techno 2012; 61(1): 455-58. doi:10.1016/j.cirp.2012.03.072. 
[8] Biller S, Meerkov S M, Yan C. Raw material release rates to ensure desired production lead time in Bernoulli serial lines. Int J Prod Res 2013; 51(14): 4349-64. DOI: 10.1080/00207543.2013.776191.

[9] Bouslah B, Gharbi A, Pellerin R. Joint optimal lot sizing and production control policy in an unreliable and imperfect manufacturing system. Int J Prod Econ 2013; 144(1): 143-56. doi:10.1016/j.ijpe.2013.01.031.

[10] Li J, Meerkov S M. Production systems engineering. Springer; 2009.

[11] Li J, Blumenfeld D E, Huang N, Alden J M. Throughput analysis of production systems: recent advances and future topics. Int J Prod Res 2009; 47(14): 3823-51. DOI: 10.1080/00207540701829752.

[12] Gershwin S B. Manufacturing systems engineering. Prentice-Hall; 1994.

[13] Liu J, Chang Q, Xiao G, Biller S. The costs of downtime incidents in serial multistage manufacturing systems. J Manuf Sci Eng 2012, 134(2): 021016. doi:10.1115/1.4005789.

[14] Biller S, Li J, Marin S P, Meerkov S M, Zhang L. Bottlenecks in Bernoulli serial lines with rework. IEEE Trans Autom Sci Eng 2010; 7(2): 208-17. 10.1109/TASE.2009.2023463.

[15] Li Y, Chang Q, Brundage M, Xiao G, Biller S. Standalone throughput analysis on the wave propagation of disturbances in production sub-systems. ASME Trans J Manuf Sci Eng 2013; 135(5): 051001. doi: 10.1115/1.4024815.

[16] Chang Q, Biller S, Xiao G. Transient analysis of downtimes and bottleneck dynamics in serial manufacturing systems. ASME Trans J Manuf Sci Eng 2010; 132(5): 051015. doi:10.1115/1.4002562.

[17] Zou J, Arinez J, Chang Q, Lei Y. Opportunity window for energy saving and maintenance in stochastic production systems. ASME Trans J Manuf Sci Eng 2016; 138(12): 121009. doi:10.1115/1.4033757.

[18] Zou J, Chang Q, Lei Y, Arinez J. Production system performance identification using sensor data. IEEE Trans Syst Man and Cybern Syst 2016 [accepted] doi:10.1109/TSMC.2016.2597062.

[19] Li J. Overlapping decomposition: a system-theoretic method for modeling and analysis of complex manufacturing systems. IEEE Trans Autom Sci Eng 2005; 2(1): 40-53. 10.1109/TASE.2004.835576.

[20] Chen C, Wang C, Zhang L, Arinez J, Xiao G. Transient performance analysis of serial production lines with geometric machines. IEEE Trans Autom Control 2015. In press. 10.1109/TAC.2015.2444071.

[21] Paul S K, Sarker R, Essam D. Real time disruption management for a two-stage batch production-inventory system with reliability considerations. Eur J Oper Res 2014; 237(1): 113-28. doi:10.1016/j.ejo-r.2014.02.005.

[22] Paul S. K, Sarker R, Essam D. Managing disruption in an imperfect production-inventory system. Comput Ind Eng 2015; 84: 101112. doi.org/10.1016/j.cie.2014.09.013.

[23] Efficiency and innovation in US manufacturing energy use, Polokwane, Limpopo: NAM, 2005.

[24] Behrendt C, Zein A, Min S. Development of an energy consumption monitoring procedure for machine tools. CIRP Ann Manuf Techn 2012; 61(1): 43-46. doi:10.1016/j.cirp.2012.03.103.

[25] Schudeleit T, Züst S, Wegener K. Methods for evaluation of energy efficiency of machine tools. Energy 2015; 93(2): 1964-70. doi:10.1016/j.energy.2015.10.074.

[26] Bujak J. Energy savings and heat efficiency in the paper industry: A case study of a corrugated board machine. Energy 2008; 33(11): 1597-08. doi:10.1016/j.energy.2008.07.005.

[27] Deif A M. A system model for green manufacturing. J Clean Prod 2011; 19(14): 1553-59. doi:10.1016/j.jclepro.2011.05.022.

[28] Figueiredo J, Martins J. Energy production system management - Renewable energy power supply integration with building automation system. Energy Convers Manage 2010; 51(6): 1120-26. doi:10.1016/j.enconman.2009.12.020.

[29] Vikhorev K, Greenough R, Brown N. An advanced energy management framework to promote energy awareness. J Clean Prod 2013; 43: 103-12. doi:10.1016/j.jclepro.2012.12.012.

[30] Duflou J R, Sutherland J W, Dornfeld D, Herrmann C, k Jeswiet J, Kara S, Hauschild M, Kellens K. Towards energy and resource efficient manufacturing: a processes and systems approach. CIRP Ann Manuf Techn 2012; 61(2): 587-09. doi:10.1016/j.cirp.2012.05.002.

[31] Brundage M P, Chang Q, Zou J, Li Y, Arinez J, Xiao G. Energy economics in the manufacturing industry: a return on investment strategy. Energy 2015; 93(2): 1426-35. doi:10.1016/j.energy.2015.10.-038.

[32] Trentesaux D. Distributed control of production systems. Eng Appl Artif Intel 2009; 22(7): 971-78. doi:10.1016/j.engappai.2009.05.001.

[33] Chung S H, Chan F T SK, Chan H K. A modified genetic algorithm approach for scheduling of perfect maintenance in distributed production scheduling. Eng Appl Artif Intel 2009; 22(7): 1005-14. doi:10.1016/j.engappai.2008.11.004.

[34] Jiao J, Xu Q, Wu Z, Ng N. Coordinating product, process, and supply chain decisions: a constraint satisfaction approach. Eng App Artif Intel 2009; 22(7): 992-1004. doi:10.1016/j.engappai.2009.02.002.

[35] Kumar P R, Varaiya P. Stochastic systems: estimation, identification, and adaptive control. Prentice-Hall; 1986.

[36] Li Y, Chang Q, Brundage M, Biller S, Arinez J, Xiao G. Market demand oriented data-driven modeling for dynamic manufacturing system control. IEEE Trans Syst Man and Cybern Syst 2014; 45(1): 109-21. 10.1109/TSMC.2014.2316268. 


\section{Figures}

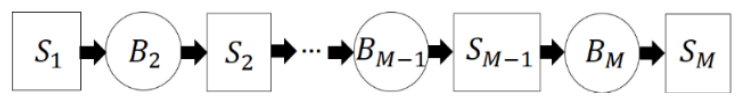

Fig. 1 General serial production line

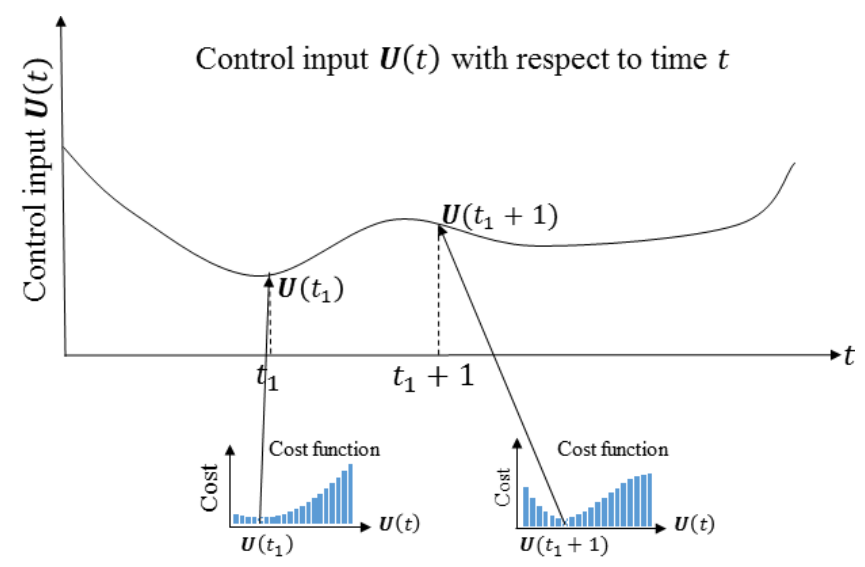

Fig. 2 Real-time control procedure

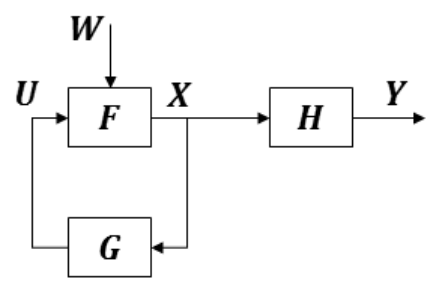

Fig. 3 Feedback control scheme for production system 


$$
S_{1} \Rightarrow B_{2} \Rightarrow S_{2} \Rightarrow B_{3} \Rightarrow S_{3} \Rightarrow B_{4} \Rightarrow S_{4} \Rightarrow B_{5} \Rightarrow S_{5} \Rightarrow B_{6} \Rightarrow S_{6}
$$

Fig 4. A six machine five buffer production system

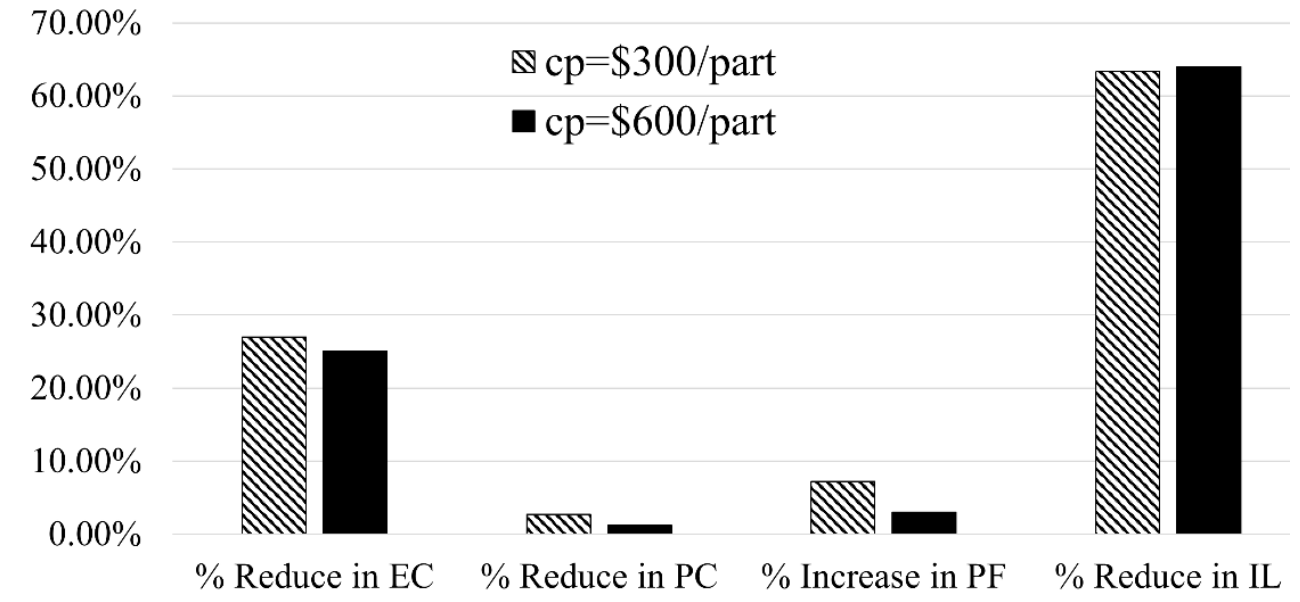

Fig. 5 Production control system improvements with $c_{p}=\$ 300 /$ part and $c_{p}=\$ 600 /$ part 


\section{Tables}

Table 1 Parameters for the six machines in the production line

\begin{tabular}{lllllll}
\hline \hline & \multicolumn{1}{c}{$S_{1}$} & \multicolumn{1}{c}{$S_{2}$} & \multicolumn{1}{c}{$S_{3}$} & \multicolumn{1}{c}{$S_{4}$} & \multicolumn{1}{c}{$S_{5}$} & \multicolumn{1}{c}{$S_{6}$} \\
\hline \hline MTBF $($ min $)$ & 5422 & 6301.2 & 11872.2 & 5440.2 & 6412.8 & 6250.8 \\
MTTR $($ min $)$ & 130.8 & 208.2 & 409.8 & 279.6 & 205.2 & 250.8 \\
Cycle time $T_{j}(\mathrm{~min})$ & 3.5 & 4.3 & 2.7 & 9.4 & 1.1 & 5.9 \\
Power rate $K_{j}(\mathrm{~kW})$ & 450 & 300 & 240 & 288 & 660 & 360 \\
\hline \hline
\end{tabular}

Table 2 Parameters for the five buffers in the production line

\begin{tabular}{llllll}
\hline \hline & $B_{2}$ & $B_{3}$ & $B_{4}$ & $B_{5}$ & $B_{6}$ \\
\hline \hline Buffer capacity & 120 & 150 & 160 & 50 & 150 \\
Initial buffer level & 70 & 30 & 50 & 40 & 50 \\
\hline \hline
\end{tabular}

Table 3 Simulation results with $c_{p}=\$ 300 /$ part

\begin{tabular}{lllll}
\hline \hline & Energy cost $(\$)$ & Production count & Profit $(\$)$ & Inventory level \\
\hline Baseline Average & 224122.5 & 3064.3 & 674644.0 & 410.5 \\
Baseline 95\% CI & {$[221674.9,226570.1]$} & {$[3031.3,3097.4]$} & {$[665225.1,684062.8]$} & {$[408.3,412.8]$} \\
Control Average & 163605.2 & 2981.8 & 723387.1 & 150.7 \\
Control 95\% CI & {$[160568.1,166642.3]$} & {$[2852.7,3110.9]$} & {$[712223.3,734551.0]$} & {$[149.3,152.2]$} \\
\hline \hline
\end{tabular}


Table 4 System improvements with distributed feedback control when $c_{p}=\$ 300 /$ part

\begin{tabular}{lllll}
\hline \hline & \% Reduce in EC & \% Reduce in PC & \% Increase in PF & \% Reduce in IL \\
\hline Average & $27.0 \%$ & $2.7 \%$ & $7.2 \%$ & $63.3 \%$ \\
\hline \hline
\end{tabular}

Table 5 Simulation results with $c_{p}=\$ 600 /$ part

\begin{tabular}{lllll}
\hline \hline & Energy cost $(\$)$ & Production count & Profit $(\$)$ & Inventory level \\
\hline Baseline Average & 224122.5 & 3064.3 & 1593936.0 & 410.5 \\
Baseline 95\% CI & {$[221674.9,226570.1]$} & {$[3031.3,3097.4]$} & {$[1574615,1613258]$} & {$[408.3,412.8]$} \\
Control Average & 167500.7 & 3027.3 & 1623468.0 & 147.9 \\
Control 95\% CI & {$[162568.1,168642.3]$} & {$[2852.7,3110.9]$} & {$[1609472,1637463]$} & {$[146.3,149.5]$} \\
\hline \hline
\end{tabular}

Table 6 System improvements with distributed feedback control when $c_{p}=\$ 600 /$ part

\begin{tabular}{lllll}
\hline \hline & \% Reduce in EC & \% Reduce in PC & \% Increase in PF & \% Reduce in IL \\
\hline Average & $25.1 \%$ & $1.2 \%$ & $3.0 \%$ & $64.0 \%$ \\
\hline \hline
\end{tabular}

